\title{
In silico Binding Predictions for Identification of HLA-DR-Promiscuous Regions and Epitopes of Mycobacterium tuberculosis Protein MPT64 (Rv1980c) and Their Recognition by Human Th1 Cells
}

\author{
Abu Salim Mustafa \\ Department of Microbiology, Faculty of Medicine, Health Sciences Centre, Kuwait University, Kuwait
}

\section{Key Words}

Mycobacterium tuberculosis $\cdot$ Rv1980c $\cdot$ In silico

predictions - HLA-DR-promiscuous regions and epitopes •

Th1 cell reactivity

\begin{abstract}
Objective: To identify HLA-promiscuous regions and epitopes of MPT64 (Rv1980c), a major secreted antigen of Mycobacterium tuberculosis, by in silico analysis for binding to HLA-DR molecules. Materials and Methods: The sequence of mature MPT64 protein (aa 1-205) was analyzed in silico for HLA-DR binding regions and T cell epitopes using ProPred, a web-based prediction server. The prediction results were experimentally verified by testing 20-mer synthetic peptides corresponding to the predicted HLA-DR binding regions and epitopes with $\mathrm{T}$ cell lines established from peripheral blood mononuclear cells of PPD-positive and HLA-heterogeneous healthy subjects in Th1 cell assays (antigen-induced proliferation and IFN- $\gamma$ secretion). Results: The in silico analysis for binding of the mature MPT64 sequence to HLA-DR molecules suggested that it could bind to molecules expressed from all HLA-DR alleles $(n=51)$ included in ProPred. Furthermore, ProPred identified 26 epitopes and 8 nonoverlapping HLA-DR binding regions (9-35 aa in length) in the Rv1980c
\end{abstract}

(C) 2010 S. Karger AG, Basel

1011-7571/10/0195-0367\$26.00/0

Fax +4161306 1234

E-Mail karger@karger.ch

www.karger.com
Accessible online at: www.karger.com/mpp sequence, with 5 regions (aa 20-44, aa 68-102, aa 132-146, aa 164-186 and aa 194-202) being HLA-DR-promiscuous. By using synthetic peptides and T cell lines in Th1 cell assays, 4 peptides of MPT64 (aa 21-40, aa 81-100, aa 171-190 and aa 191-20), from 4 of the 5 HLA-DR-promiscuous regions predicted by ProPred, were experimentally verified to be HLADR-promiscuous and to have immunodominant epitopes. Conclusion: The in silico method (ProPred) suggested promiscuous HLA-DR-binding of mature MPT64 and identified HLA-promiscuous and immunodominant regions and epitopes of this protein.

Copyright @ 2010 S. Karger AG, Basel

\section{Introduction}

Tuberculosis (TB) is a major infectious disease of worldwide prevalence. About one third of the world population is infected with Mycobacterium tuberculosis, 9.3 million people develop active TB, and 1.8 million people die of TB each year [1]. The current efforts to reduce the global problem of TB have been focused on improved diagnosis and effective chemotherapy; however, these efforts have not been effective in reducing the worldwide problem of TB [1]. Therefore, improved diagnostic and 
therapeutic efforts need to be combined with additional preventive efforts. Hence, the development of effective vaccines to prevent the development of active disease is considered a major goal to control the worldwide epidemic of TB [2].

A primary criterion to identify antigens/peptides of M. tuberculosis as potential vaccine candidates against TB is their recognition by Th1 cells, which are the major players in protective immunity against TB [3-5]. It has previously been shown that MPT64 (Rv1980c), a major secreted antigen of $M$. tuberculosis, is recognized by Th1 cells from M. bovis BCG-vaccinated and M.-tuberculosisinfected healthy subjects $[6,7]$, and thus could be useful as a candidate vaccine against TB. However, human Th1 cells recognize mycobacterial protein/peptide antigens in association with human leukocyte antigen (HLA) class II molecules and, in particular, in association with highly polymorphic HLA-DR molecules [8-12]. To be useful in HLA-heterogeneous human populations, it is imperative that a candidate protein/peptide vaccine or diagnostic antigen is able to bind HLA-DR molecules promiscuously, i.e. to several commonly expressed HLA-DR molecules [13-15]. Several antigenic proteins proposed as candidate vaccines against TB and/or diagnostic antigens, e.g. ESAT-6, CFP10, Ag85B, MPT70 or MPT63, have been characterized for HLA-DR binding by in silico prediction using the ProPred server [16], and found promiscuous HLA-DR binders both with respect to full-length proteins and immunodominant epitopes [17-19]. To our knowledge, the MPT64 sequence has not been characterized for HLA-DR binding prediction and identification of epitope regions using ProPred. Furthermore, the analysis of such data in relation to actual presentation of Rv1980c and the predicted regions and epitopes to Th1 cells from healthy subjects is also lacking.

In this study, the Rv1980c sequence was analyzed in silico for binding to molecules expressed from 51 HLADR alleles included in ProPred, and regions predicted to be HLA-DR binders were evaluated for HLA-promiscuous Th1 cell reactivity using synthetic peptides and T cell lines established from PPD-positive and HLA-DR-typed healthy subjects.

\section{Materials and Methods}

In silico Analysis of MPT64 for HLA-DR Binding Regions and Identification of T Cell Epitopes

The primary structure of the mature MPT64 sequence was first analyzed for T cell epitopes by the ProPred method for binding to HLA-DR molecules using the server (http://www.imtech. res.in/raghava/propred/) [16]. The ProPred analysis was performed at the default setting, i.e. threshold value of 3.0. The peptides predicted to bind $>50 \%(\geq 5 / 9)$ serologically defined HLADR molecules included in ProPred were considered promiscuous for binding [18].

\section{Synthetic Peptides}

Synthetic peptides (20-mers; overlapping with neighboring peptides by 10 aa, if more than 1 peptide was required to cover the HLA-DR binding region) spanning the sequence of the ProPred-predicted HLA-DR binding regions of MPT64 were purchased from Thermo Hybaid GmbH, Ulm, Germany. These peptides were synthesized using fluonerylmethoxycarbonyl chemistry, as described previously $[20,21]$. The stock concentrations $(5 \mathrm{mg} / \mathrm{ml})$ of the peptides were prepared in normal saline $(0.9 \%)$ by vigorous pipetting, and the working concentrations were prepared by further dilution in tissue culture medium RPMI-1640 (Life Technologies, Paisley, UK) [22].

\section{Experimental Verification of ProPred-Predicted Epitopes in}

Th1 cell Assays

The synthetic peptides were experimentally verified for Th1 cell reactivity by testing $T$ cell lines, established from HLA-heterogeneous healthy humans, in peptide-induced proliferation and IFN- $\gamma$ secretion assays using procedures described previously [23]. For proliferation assays, the results were calculated as stimulation index, which is defined as cpm in peptide-stimulated cultures/cpm in cultures without peptide, and stimulation indices of $\geq 2$ were considered positive [24]. Secretion of IFN- $\gamma$ in response to a given peptide was considered positive with $\mathrm{E} / \mathrm{C}>2$ (E/C $=$ IFN- $\gamma$ concentration in cultures stimulated with peptide/ IFN- $\gamma$ concentration in cultures without peptide) and IFN- $\gamma$ concentration $\geq 1.5 \mathrm{IU} / \mathrm{ml}$ [24]. The Th1 cell responses were considered strong, moderate and weak with peptides showing positive responses with $\mathrm{T}$ cell lines from $>60,30-60$ and $<30 \%$ donors, respectively [25].

Informed consent was obtained from all the subjects included in the study, and the experimental protocol was approved by the Ethics Committee, Faculty of Medicine, Kuwait University, Kuwait.

\section{Results}

The ProPred analysis of the mature MPT64 sequence (aa 1-205) showed that this protein was predicted to bind all serologically defined HLA-DR molecules expressed from 51 (100\%) HLA-DR alleles included in the ProPred graphical web tool (table 1), and it had a total of 26 epitopes (9-mers each), which were scattered throughout the protein sequence (table 2). In addition, a total of 8 nonoverlapping regions from 9 to 35 aa in length (aa 9-17, aa 20-44, aa $46-62$, aa $68-102$, aa $115-127$, aa $132-146$, aa $164-186$ and aa 194-202) were predicted to bind HLA-DR alleles with binding frequencies ranging from $2 / 51$ (4\%) to $47 / 51$ (92\%) (table 1). Five of these regions, i.e. region 2 (aa 20 44), region 4 (aa 68-102), region 6 (aa 132-146), region 7 
Table 1. ProPred analysis for HLA-DR binding regions of MPT64 to 51 alleles of 9 serologically defined HLA-DR molecules

\begin{tabular}{|c|c|c|c|c|c|c|c|c|c|c|c|c|}
\hline \multirow{2}{*}{\multicolumn{2}{|c|}{$\frac{\text { Binding region }}{\text { No. sequence }}$}} & \multicolumn{9}{|c|}{ Binding to HLA-DR molecules } & \multirow{2}{*}{$\frac{\text { All alleles }}{\mathrm{P} / \mathrm{T}}$} & \multirow{2}{*}{$\frac{\text { All molecules }}{\mathrm{P} / \mathrm{T}}$} \\
\hline & & B1.1 & B1.3 & B1.4 & B1.7 & B1.8 & B1.11 & B1.13 & B1.15 & B5.1 & & \\
\hline 1 & aa 9-17 & $0 / 2$ & $0 / 7$ & $2 / 9$ & $0 / 2$ & $0 / 6$ & $0 / 9$ & $0 / 11$ & $0 / 3$ & $0 / 2$ & $2 / 51(4)$ & $1 / 9(11)$ \\
\hline 2 & aa $20-44$ & $2 / 2$ & $7 / 7$ & $9 / 9$ & $0 / 2$ & $6 / 6$ & $9 / 9$ & $11 / 11$ & $3 / 3$ & $0 / 2$ & $47 / 51(92)$ & $7 / 9(78)$ \\
\hline 3 & aa $46-62$ & $0 / 2$ & $1 / 7$ & $5 / 9$ & $0 / 2$ & $0 / 6$ & $1 / 9$ & $1 / 11$ & $0 / 3$ & $0 / 2$ & $8 / 51(16)$ & $4 / 9(44)$ \\
\hline 4 & aa $68-102$ & $1 / 2$ & $1 / 7$ & $9 / 9$ & $0 / 2$ & $5 / 6$ & $3 / 9$ & $2 / 11$ & $2 / 3$ & $2 / 2$ & $25 / 51(49)$ & $8 / 9(89)$ \\
\hline 5 & aa $115-127$ & $0 / 2$ & $1 / 7$ & $2 / 9$ & $0 / 2$ & $0 / 6$ & $1 / 9$ & $0 / 11$ & $0 / 3$ & $0 / 2$ & $4 / 51(8)$ & $3 / 9(33)$ \\
\hline 6 & aa $132-146$ & $0 / 2$ & $5 / 7$ & $3 / 9$ & $0 / 2$ & $2 / 6$ & $4 / 9$ & $2 / 11$ & $0 / 3$ & $0 / 2$ & $16 / 51(31)$ & $5 / 9(56)$ \\
\hline 7 & aa $164-186$ & $2 / 2$ & $7 / 7$ & $4 / 9$ & $2 / 2$ & $3 / 6$ & $2 / 9$ & $3 / 11$ & $3 / 3$ & $0 / 2$ & $26 / 51(51)$ & $8 / 9(89)$ \\
\hline 8 & aa $194-202$ & $0 / 2$ & $4 / 7$ & $1 / 9$ & $0 / 2$ & $6 / 6$ & $9 / 9$ & $11 / 11$ & $2 / 3$ & $0 / 2$ & $33 / 51(65)$ & $6 / 9(67)$ \\
\hline
\end{tabular}

Binding $=$ Number of alleles predicted to bind/number of alleles included; $\mathrm{P} / \mathrm{T}=$ number positive/number tested; figures in parentheses are percentages.

${ }^{1}$ The values showing promiscuous binding to HLA-DR molecules (as defined in 'Materials and Methods') are given in italics.

Table 2. Comparison of the number of predicted epitopes and HLA-DR binding frequency with the responses of T cell lines to peptides corresponding to HLA-DR binding regions in Th1 cell assays

\begin{tabular}{|c|c|c|c|c|c|c|}
\hline \multirow[t]{2}{*}{$\begin{array}{l}\text { Region } \\
\text { No. }\end{array}$} & \multirow[t]{2}{*}{ Sequence of peptide(s) covering the region ${ }^{1}$} & \multirow[t]{2}{*}{ Epitopes } & \multicolumn{2}{|c|}{$\begin{array}{l}\text { Binding to HLA-DR } \\
\text { molecules }^{2}\end{array}$} & \multicolumn{2}{|c|}{$\begin{array}{l}\text { Responders in } \\
\text { Th1 cell assays }\end{array}$} \\
\hline & & & $\mathrm{P} / \mathrm{T}$ & $\%$ & $\mathrm{P} / \mathrm{T}$ & $\%$ \\
\hline 1 & 1-APKTYCEELKGTDTGQACQI-20 & 1 & $1 / 9$ & 11 & $0 / 14$ & 0 \\
\hline 2 & 11-GTDTGQACQIQMSDPAYNINISLPSYYPDQKSLENYIAQT-50 & 4 & $7 / 9$ & 78 & $11 / 14$ & 79 \\
\hline 3 & 41-KSLENYIAQTRDKFLSAATSSTPREAPYEL-70 & 2 & $4 / 9$ & 44 & $3 / 14$ & 21 \\
\hline 6 & 131-PLPVVFPIVQGELSKQTGQQ-150 & 3 & $5 / 9$ & 56 & $4 / 14$ & 29 \\
\hline 7 & 161-PVNYQNFAVTNDGVIFFFNPGELLPEAAGP-190 & 6 & $8 / 9$ & 89 & $9 / 14$ & 64 \\
\hline 8 & 191-TQVLVPRSAIDSMLA-205 & 1 & $6 / 9$ & 67 & $9 / 14$ & 64 \\
\hline
\end{tabular}

$\mathrm{P} / \mathrm{T}=$ Number positive/number tested.

${ }^{1}$ The sequences predicted to bind HLA-DR molecules are underlined and the obligatory anchor (starting) residues are marked in bold. ${ }^{2}$ The values showing promiscuous binding to HLA-DR molecules (as defined in 'Materials and Methods') are given in italics. ${ }^{3}$ The values showing strong responses (as defined in 'Materials and Methods') in Thl cell assays (antigen-induced proliferation and/or IFN- $\gamma$ secretion) are given in italics.

(aa 164-186) and region 8 (aa 194-202), were predicted to be promiscuous HLA-DR binders (table 1). Furthermore, 21 of 26 epitopes were present in these HLA-DR-promiscuous regions with 4 regions having more than 1 overlapping epitope of 9 aa each, i.e. 4 epitopes in region 2 (aa $20-29$, aa $27-35$, aa $29-37$ and aa $36-44), 7$ epitopes in region 4 (aa 68-76, aa 70-78, aa 77-85, aa 81-89, aa 89-97, aa 91-99 and aa 94-102), 3 epitopes in region 6 (aa 132140, aa 134-142 and aa 138-156) and 6 epitopes in region 7 (aa $164-172$, aa $169-177$, aa $174-182$, aa $175-183$, aa $176-$ 184 and aa 178-186), whereas HLA-DR-promiscuous region 8 had only 1 epitope (aa 194-202) (table 2).

In silico Binding Predictions for Th1 Cell Epitopes of Rv1980c
Testing of synthetic peptides with $\mathrm{T}$ cell lines showed that 4 of the 5 HLA-DR-promiscuous regions, i.e. regions 2, 4, 7 and 8 , induced strong Th1 cell responses, whereas peptides belonging to all of the 3 non-HLA-DR-promiscuous regions, i.e. regions 1,3 and 5, induced nil to weak responses in Th1 cell assays (table 2 ).

Among the 4 HLA-DR-promiscuous and strong Th1 cell-stimulating regions, only region 8 was represented by a single peptide, whereas the other 3 regions required more than 1 peptide to cover the sequences, i.e. 3 peptides for region 2, 4 peptides for region 4 and 2 peptides for region 7 (table 3). These peptides were further analyzed for 
Table 3. Comparison of the number of predicted epitopes and HLA-DR binding frequency of peptides of regions 2 , 4 and 6 with Th1 cell responses of $\mathrm{T}$ cell lines

\begin{tabular}{|c|c|c|c|c|c|c|}
\hline \multirow[t]{2}{*}{$\begin{array}{l}\text { Region } \\
\text { No. }\end{array}$} & \multirow[t]{2}{*}{ Peptide sequence ${ }^{1}$} & \multirow[t]{2}{*}{ Epitopes } & \multicolumn{2}{|c|}{$\begin{array}{l}\text { Binding to HLA-DR } \\
\text { molecules }^{2}\end{array}$} & \multicolumn{2}{|c|}{$\begin{array}{l}\text { Responders in Th1 cell } \\
\text { assays }^{3}\end{array}$} \\
\hline & & & $\mathrm{P} / \mathrm{T}$ & $\%$ & $\mathrm{P} / \mathrm{T}$ & $\%$ \\
\hline \multirow[t]{3}{*}{2} & 11-GTDTGQACQIQMSDPAYNIN-30 & 1 & $4 / 9$ & 44 & $2 / 14$ & 14 \\
\hline & 21-QMSDPAYNINISLPSYYPDQ-40 & 2 & $7 / 9$ & 78 & $8 / 14$ & 57 \\
\hline & 31-ISLPSYYPDQKSLENYIAQT-50 & 1 & $2 / 9$ & 22 & $3 / 14$ & 21 \\
\hline \multirow{3}{*}{4} & 71-NITSATYQSAIPPRGTQAVV-90 & 2 & $5 / 9$ & 56 & $2 / 14$ & 14 \\
\hline & 81-IPPRGTQAVVLKVYQNAGGT-100 & 3 & $6 / 9$ & 67 & $8 / 14$ & 57 \\
\hline & 90-LKVYQNAGGTHPTTTYKAFD-110 & 2 & $4 / 9$ & 44 & $3 / 14$ & 21 \\
\hline \multirow[t]{2}{*}{7} & 161-PVNYYQNFAVTNDGVIFFFNP-180 & 2 & $5 / 9$ & 56 & $3 / 14$ & 21 \\
\hline & 171-NDGVIFFFNPGELLPEAAGP-190 & 4 & $6 / 9$ & 67 & $9 / 14$ & 64 \\
\hline
\end{tabular}

$\mathrm{P} / \mathrm{T}=$ Number positive/number tested.

${ }^{1}$ The sequences predicted to bind HLA-DR molecules are underlined and the obligatory anchor (starting) residues are marked in bold. ${ }^{2}$ The values showing promiscuous binding to HLA-DR molecules (as defined in 'Materials and Methods') are given in italics. ${ }^{3}$ The values showing moderate to strong responders (as defined in 'Materials and Methods') in Th1 cell assays (antigen-induced proliferation and/or IFN- $\gamma$ secretion) are given in italics.

HLA-DR binding prediction and number of epitopes, which were correlated with the responses of $\mathrm{T}$ cell lines in Th1 cell assays (table 3 ). The results showed that the peptides aa $21-40$, aa $81-100$ and aa $171-190$, of regions 2,4 and 7 , respectively, showed the highest binding predictions to HLA-DR molecules, had the highest number of epitopes, and were the best stimulators of Th1 cells (table 3).

A further analysis of the predicted HLA-DR binding regions and peptides of MPT64 for Th1 cell responses in relation to expression of HLA-DR molecules by the donors of $\mathrm{T}$ cell lines confirmed the promiscuous presentation of the peptides aa $21-40$, aa $81-100$, aa $171-190$ and aa 191-205 because these peptides were recognized by $\mathrm{T}$ cell lines from donors with varied HLA-DR types, i.e. HLA-DR 3, 4, 5, 6, 7, 13, 52 and 53 (table 4).

\section{Discussion}

MPT64 (Rv1980c) is among the major secreted protein antigens of M. tuberculosis, and like other secreted proteins of the pathogen, which are recognized by protective Th1 cells in humans, e.g. ESAT-6, CFP10, Ag85B, MPT70 or MPT63 [17-19], this protein is also recognized by human Th1 cells $[6,7]$ and thus could be useful in the diagnosis of or included among the new candidate vaccines against TB. However, any protein as a vaccine or diagnostic antigen for use in humans must be able to bind several HLA-DR molecules so that it is presented to Th1 cells in a HLA-promiscuous manner, a requirement for any new vaccine or antigen to be useful in HLA-heterogeneous human populations [13-15]. All of the other secreted proteins of $M$. tuberculosis mentioned above have previously been analyzed for HLA binding prediction using the ProPred server [19-21]; however, to our knowledge, such an analysis for MPT64 has been performed for the first time in this study.

The HLA-DR binding analysis of the MPT64 sequence by ProPred suggests that there are 8 nonoverlapping regions scattered throughout the protein sequence, which could bind to HLA-DR alleles with frequencies ranging from 4 to $92 \%$, and the complete mature protein could bind to all (51/51, 100\%) HLA-DR alleles of 9 frequently expressed and serologically defined HLA-DR molecules included in the ProPred [16]. Although none of the HLADR binding regions were predicted to bind all the alleles by themselves, the complete sequence of MPT64 was predicted to bind all the molecules expressed from 51 HLADR alleles included in the ProPred, because of differential binding of the regions to various HLA-DR alleles. Furthermore, 5 of the 9 HLA-DR binding regions are predicted to be promiscuous HLA-DR binders, because these regions were predicted to bind $>50 \%$, i.e. $>5 / 9$, serologi- 
Table 4. Comparison of HLA-DR binding prediction of peptides of MPT64 using ProPred with responders in Th1 cell assays and HLA-DR types of responding donors

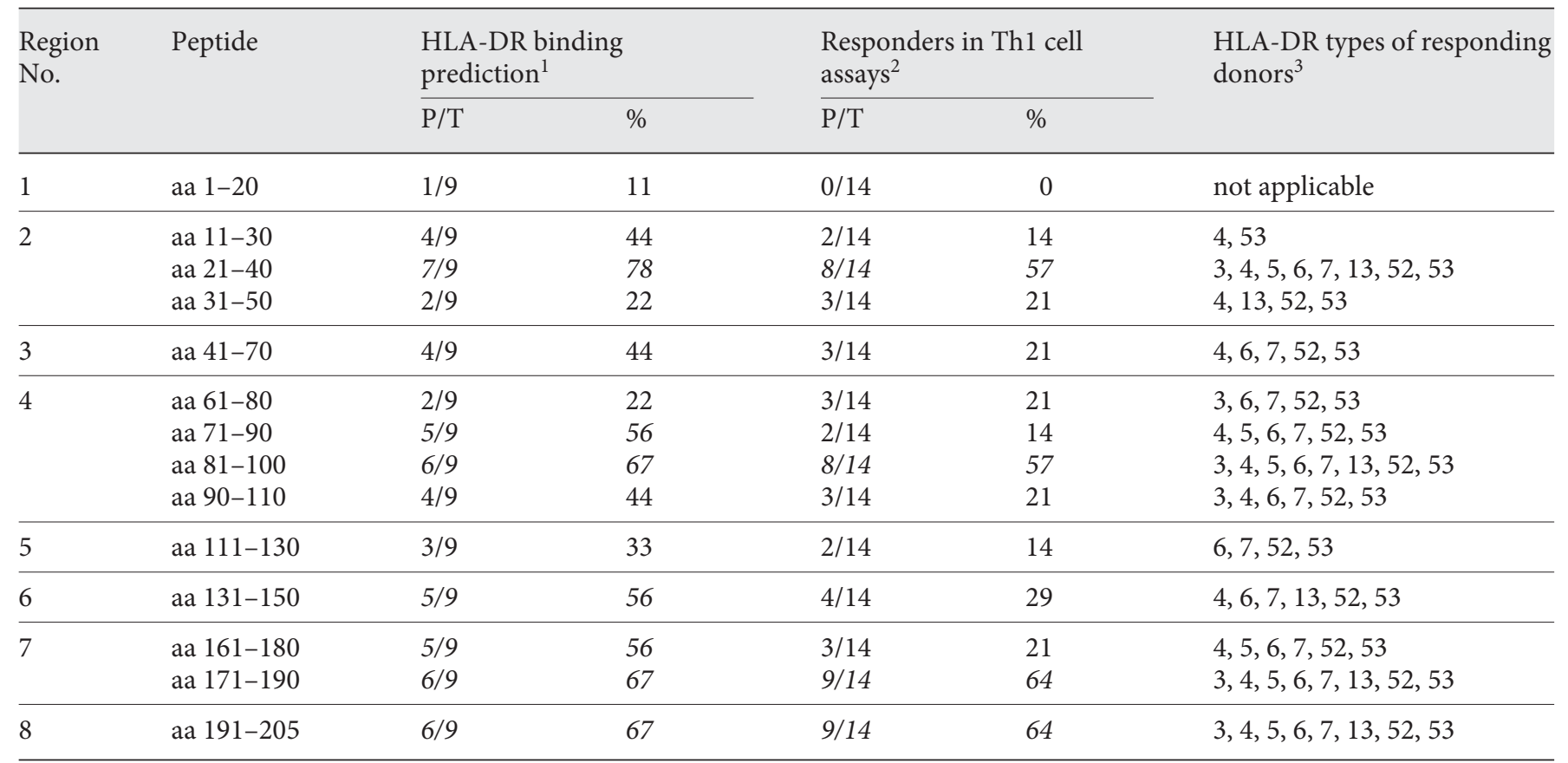

$\mathrm{P} / \mathrm{T}=$ Number positive/number tested.

${ }^{1}$ The values showing promiscuous binding to HLA-DR molecules (as defined in 'Materials and Methods') are given in italics. ${ }^{2}$ The values showing moderate to strong responders (as defined in 'Materials and Methods') in Th1 cell assays (antigen-induced proliferation and/or IFN- $\gamma$ secretion) are given in italics. ${ }^{3}$ The donors of T cell lines used for Th1 cell assays were positive for HLA-DR 3, 4, 5, $6,7,13,52$ and 53 molecules.

cally defined HLA-DR molecules included in the ProPred $[18,19]$. In addition to being promiscuous for HLA-DR binding, the ProPred analysis also predicted that MPT64 has a large number of Th1 cell epitopes scattered throughout its sequence. These results are compatible with previous findings of the recognition of MPT64 and a large number of its peptides by peripheral blood mononuclear cells and T cell lines from HLA-heterogeneous donors in Th1 cell assays $[6,7]$. Thus, the present results reinforce the highly promiscuous nature of MPT64 for presentation to Th1 cells and further support the use of this protein in vaccine or diagnostic applications.

In addition to prediction of HLA-DR binding regions by ProPred, we have also evaluated the Th1 cell reactivity of these regions using 20-mer synthetic peptides. Because these regions were of varying length, i.e. 9-35 aa, 4 regions required more than 1 peptide to cover their sequence, i.e. region 2 ( 3 peptides), region 3 ( 2 peptides), region 4 (4 peptides) and region 7 ( 2 peptides). The evaluation of all regions by using synthetic peptides in Th1 cell assays showed that 4 of the 5 regions predicted as HLADR promiscuous by ProPred were also strong stimulators of Th1 cell reactivity among $\mathrm{T}$ cell lines from donors with varied HLA-DR types. The reason for 1 of the 5 predicted HLA-DR-promiscuous regions, i.e. region 6, not showing strong Th1 cell reactivity could be due to its relatively lower level of binding to HLA-DR alleles $(16 / 51,31 \%)$ compared to other regions (from 25/51 to 47/51, 49-92\%), which showed strong Th1 cell reactivity. Alternatively, Th1 cells capable of recognizing the epitopes of region 6 may have been lacking in the tested individuals, as has been reported previously for some other mycobacterial proteins [11, 17-19]. Further analyses of HLA-DR-promiscuous regions with strong Th1 cell reactivity, which required more than 1 peptide to cover their sequence, was performed with individual peptides to identify the HLADR-promiscuous and immunodominant peptides. The results show that for each region, the immunodominant peptides had the highest numbers of $\mathrm{T}$ cell epitopes and were HLA-DR-promiscuous for binding by ProPred. 
Thus, except for one region and its peptide showing discordant results, all other regions and peptides showed concordance in terms of HLA-DR binding prediction by ProPred and response in Th1 cell assays with $\mathrm{T}$ cell lines from donors of varied HLA-DR types.

\section{Conclusion}

The in silico analysis suggests that MPT64 has several HLA-DR-promiscuous regions and a large number of $\mathrm{T}$ cell epitopes scattered throughout the sequence of this protein. Furthermore, strong recognition of HLA-DRpromiscuous regions by Th1 cells supports the notion that MPT64 deserves consideration for inclusion in diagnostic applications or as a candidate vaccine against human TB.

\section{Acknowledgments}

This study was supported by Kuwait University Research Administration grant MI06/08. The buffy coats from the donors were provided by the Central Blood Bank, Kuwait.

\section{References}

1 Donald PR, van Helden PD: The global burden of tuberculosis - combating drug resistance in difficult times. N Engl J Med 2009; 360:2393-2395

2 Mustafa AS: Vaccine potential of Mycobacterium tuberculosis-specific genomic regions: in vitro studies in humans. Expert Rev Vaccines 2009;8:1309-1312.

3 Mustafa AS: Development of new vaccines and diagnostic reagents against tuberculosis. Mol Immunol 2002;39:113-119.

4 Al-Attiyah R, Mustafa AS, Abal AT, Madi NM, Andersen P: Restoration of mycobacterial antigen-induced proliferation and interferon-gamma responses in peripheral blood mononuclear cells of tuberculosis patients upon effective chemotherapy. FEMS Immunol Med Microbiol 2003;38:249-256.

5 Al-Attiyah R, El-Shazly A, Mustafa AS: Assessment of in vitro immunity to Mycobacterium tuberculosis in a human peripheral blood infection model using a luciferase reporter construct of $M$. tuberculosis H37Rv. Clin Exp Immunol 2006; 145:520-527.

6 Mustafa AS: HLA-promiscuous Th1-cell reactivity of MPT64 (Rv1980c), a major secreted antigen of Mycobacterium tuberculosis, in healthy subjects. Med Princ Pract 2009;18: 385-392.

7 Mustafa AS, Shaban F: Mapping of Th1-cell epitope regions of Mycobacterium tuberculosis protein MPT64 (Rv1980c) using synthetic peptides and T-cell lines from M.-tuberculosis-infected healthy humans. Med Princ Pract 2010;19:122-128.

8 Mustafa AS: HLA-restricted immune response to mycobacterial antigens: relevance to vaccine design. Hum Immunol 2000;61: $166-171$.

9 Mustafa AS: Biotechnology in the development of new vaccines and diagnostic reagents against tuberculosis. Curr Pharm Biotechnol 2001;2:157-173.

10 Al-Attiyah R, Shaban FA, Wiker HG, Oftung F, Mustafa AS: Synthetic peptides identify promiscuous human Th1 cell epitopes of the secreted mycobacterial antigen MPB70. Infect Immun 2003;71:1953-1960.
11 Al-Attiyah R, Mustafa AS: Computer-assisted prediction of HLA-DR binding and experimental analysis for human promiscuous Th1 cell peptides in a novel $24-\mathrm{kDa}$ secreted lipoprotein (LppX) of Mycobacterium tuberculosis. Scand J Immunol 2004;59:16-24.

-12 Mustafa AS, Shaban FA, Al-Attiyah R, Abal AT, El-Shamy AM, Andersen P, Oftung F: Human Th1 cell lines recognize the Mycobacterium tuberculosis ESAT- 6 antigen and its peptides in association with frequently expressed HLA class II molecules. Scand J Immunol 2003;57:125-134

13 Mustafa AS: Progress towards the development of new anti-tuberculosis vaccines; in Smith LT (ed): Focus on Tuberculosis Research. New York, Nova Science Publishers, 2005, pp 47-76.

14 Mustafa AS: Recombinant and synthetic peptides to identify Mycobacterium tuberculosis antigens and epitopes of diagnostic and vaccine relevance. Tuberculosis (Edinb) 2005;85:367-376.

15 Mustafa AS: Mycobacterial gene cloning and expression, comparative genomics, bioinformatics and proteomics in relation to the development of new vaccines and diagnostic reagents. Med Princ Pract 2005; 14(suppl 1): 27-34.

16 Singh H, Raghava GPS: ProPred: prediction of HLA-DR binding sites. Bioinformatics 2001;17:1236-1237.

17 Mustafa AS, Abal AT, Shaban F, El-Shamy AM, Amoudy HA: HLA-DR binding prediction and experimental evaluation of mycolyltransferase (Ag85B), a major secreted antigen of Mycobacterium tuberculosis. Med Princ Pract 2005; 14:140-146.

-18 Mustafa AS, Shaban FA: ProPred analysis and experimental evaluation of promiscuous Th1 cell epitopes of three major secreted antigens of Mycobacterium tuberculosis. Tuberculosis (Edinb) 2006;86:115-124.
19 Mustafa AS: Th1-cell reactivity and HLADR binding prediction for promiscuous recognition of MPT63 (Rv1926c), a major secreted protein of Mycobacterium tuberculosis. Scand J Immunol 2009;69:213-222.

20 Mustafa AS, Al-Attiyah R, Hanif SNM, Shaban FA: Efficient testing of large pools of $M y$ cobacterium tuberculosis RD1 peptides and identification of major antigens and immunodominant peptides recognized by human Th1 cells. Clin Vaccine Immunol 2008; 15 : 916-924.

-21 Hanif SNM, El-Shamy AM, Al-Attiyah R, Mustafa AS: Whole blood assays to identify Th1 cell antigens and peptides encoded by Mycobacterium-tuberculosis-specific RD1 genes. Med Princ Pract 2008;17:244249 .

22 Al-Attiyah R, Mustafa AS: Characterization of human cellular immune responses to novel Mycobacterium tuberculosis antigens encoded by genomic regions absent in $\mathrm{Myco}$ bacterium bovis BCG. Infect Immun 2008; 76:4190-4198.

-23 Mustafa AS, Shaban FA, Abal AT, Al-Attiyah R, Wiker HG, Lundia KEA, Oftung F, Huygen $\mathrm{K}$ : Identification and HLA restriction of naturally derived Th1-cell epitopes from the secreted Mycobacterium tuberculosis antigen $85 \mathrm{~B}$ recognized by antigen-specific human CD4+ T cell lines. Infect Immun 2000; 68:3933-3940.

24 Mustafa AS, El-Shamy AM, Madi NM, Amoudy HA, Al-Attiyah R: Cell-mediated immune responses to complex and single mycobacterial antigens in tuberculosis patients with diabetes. Med Princ Pract 2008; 17:325-330.

25 Al-Attiyah RJ, Mustafa AS: Mycobacterial antigen-induced $\mathrm{T}$ helper type 1 (Th1) and Th2 reactivity of peripheral blood mononuclear cells from diabetic and non-diabetic tuberculosis patients and Mycobacterium bovis bacilli Calmette-Guérin (BCG)-vaccinated healthy subjects. Clin Exp Immunol 2009; 158:64-73. 